\title{
Value of cancer antigen 125 for diagnosis of pleural endometriosis in females with
} recurrent pneumothorax

\author{
P. Bagan*, , P. Berna*, J. Assouad*, V. Hupertan\#, F. Le Pimpec Barthes* \\ and M. Riquet*
}

ABSTRACT: The thorax is the most frequent extrapelvic location of endometriosis. Thoracic endometriosis is probably responsible for the high rate of recurrent pneumothoraces in females. The goal of the present prospective study was to assess the value of cancer antigen (CA) 125 measurement in the detection of endometriosis in order to further enable early and adequate treatment of catamenial pneumothorax.

Between January 2004 and March 2006, 31 females (mean age 32 yrs) underwent pneumothorax surgery. The control group comprised 17 males (mean age $27 \mathrm{yrs),} \mathrm{who} \mathrm{underwent}$ videothoracoscopic pleural abrasion. Serum CA125 was measured around a menstrual period in females and before surgery in males.

Videothoracoscopically diagnosed endometriosis occurred in $29 \%$ of females. The CA125 concentration was significantly higher in females with endometriosis compared to disease-free females (76.1 versus $\left.16 \mathrm{U} \cdot \mathrm{mL}^{-1}\right)$. The mean value in males was similar to that observed in diseasefree females.

The frequency of thoracic endometriosis-related pneumothorax corresponds to, on average, a third of females presenting with recurrent pneumothorax. Early detection can be achieved with serum cancer antigen 125 measurement and may be helpful in indicating videothoracoscopic surgery.

KEYWORDS: Cancer antigen 125, catamenial pneumothorax, thoracic endometriosis

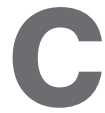
atamenial pneumothorax $(\mathrm{CP})$ is an entity of recurring spontaneous pneumothorax in females during their reproductive years. It has been associated with thoracic endometriosis (TE) but its physiological mechanism remains unclear. Traditional surgical pleurodesis is associated with a high rate of recurrence. In 2004, KOROM et al. [1] outlined the frequent lack of recognition of TE. The mean \pm SD number of recurrences before definitive diagnosis and treatment was $5.1 \pm 6.0$ (several reports listed $>30$ recurrences before treatment [2-4]). Indeed, when TE is investigated in females of reproductive age with a spontaneous pneumothorax, even when the pneumothorax occurs outside the menstrual period, the mean rate of TE is 25$30 \%[5,6]$.

TsUNEZUKA et al. [7] observed that immunohistochemical staining of endometrial cells on partial resection of the diaphragm showed antibodies directed against cancer antigen (CA)125 in cases of CP. The authors observed that serum CA125 level, which was very high before operation, decreased to within the normal range after diaphragmatic resection. In a recent report, KAFALI et al. [8] managed to successfully diagnose pelvic endometriosis by measurement of CA125 level during menstruation. Similarly, the aim of the present study was to detect endometriosis in females with recurrent pneumothorax. Thus, the goal of this prospective study was to evaluate serum CA125 level in the diagnosis of TE in females of reproductive age presenting with recurrent spontaneous pneumothorax, and hence to facilitate adequate surgical and hormonal treatment.

\section{MATERIAL AND METHODS}

\section{Patients}

Between January 2004 and March 2006, 31 consecutive females referred to the Georges Pompidou European Hospital (Paris V University, Paris, France) for surgery involving primary spontaneous pneumothorax were included in the present study. Their mean age

\section{AFFILIATIONS}

*Dept of Thoracic Surgery, Georges Pompidou European Hospital, Paris V University, Paris, and

${ }^{\#}$ Dept of Thoracic, Vascular Surgery and Urology, Surgery Clinic, Victor

Dupouy Hospital, Argenteuil, France.

CORRESPONDENCE

M. Riquet

Service de Chirurgie Thoracique

Hôpital Européen Georges Pompidou

20-40 rue Leblanc

75908 Paris

France

Fax: 33156093380

E-mail: marc.riquet@hop.egp.aphop-paris.fr

Received:

June 182006

Accepted after revision:

August 302007

STATEMENT OF INTEREST

None declared. 
was 32 yrs (range 19-51 yrs). Investigation of gynaecological history was performed before each surgical procedure. Indications for surgery were recurrent pneumothorax (two or more episodes; $n=21)$, persistent air leakage $(n=8)$, spontaneous haemopneumothorax $(n=1)$ and post-operative recurrence $(n=1)$. A control group was chosen in order to avoid the possible perturbations of CA125 level caused by pleural effusion [9]. The control group comprised 17 males with primary spontaneous recurrent pneumothorax, who underwent videothoracoscopic pleural abrasion during the beginning of the study period. The mean age of the control group was 27 yrs (range 16-56 yrs).

All patients gave informed consent and the study was approved by the ethics committee of the Institute of Clinical Research (Georges Pompidou European Hospital).

CA125 was prospectively measured in all females around one menstrual period (range 2 days before to 2 days after), when its concentration is at its highest. CA125 levels were measured before surgery in males. In female patients with a pneumothorax occurring outside the menstrual period, CA125 levels were measured at the beginning of the next cycle in the outpatient clinic of the Georges Pompidou European Hospital. CA125 levels were considered normal at $<35 \mathrm{U} \cdot \mathrm{mL}^{-1}$ (hospital laboratory reference value).

All patients underwent videothoracoscopy. Resection of blebs or bullae was performed systematically with a stapler. The diaphragmatic surface was examined in the females. Diaphragmatic biopsy procedures were performed each time defects and nodules were observed. In these cases, a technique performed by the current authors since 1990 [10] was applied; the treatment consisted of the insertion of a polyglactin mesh (Vicryl; Ethicon, Inc., Sommerville, NJ, USA) to cover the tendinous part of the diaphragm. The meshes were held in place with endostaples.

\section{Statistical analysis}

All measurements are presented as mean \pm SD. Mean serum CA125 concentrations were compared using a two-sample

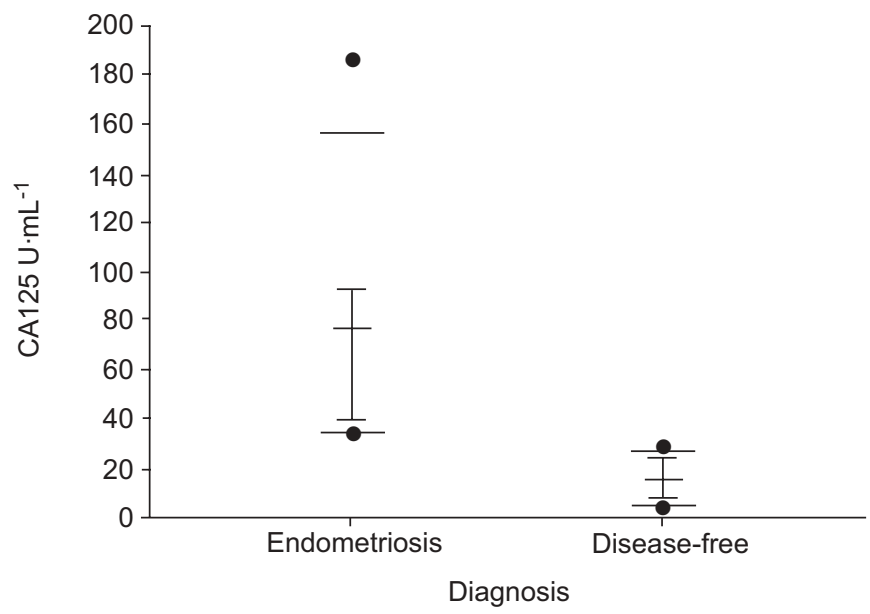

FIGURE 1. Comparison of cancer antigen (CA)125 level according to diaphragmatic and pleural pathological examination in females $(p<0.0001)$. Data are presented as mean and 95\% confidence interval ( $\bullet$ outliers) t-test for between-group comparisons and a paired t-test for within-group comparisons. A p-value of $<0.05$ was considered significant. Optimum cut-off points for use in a predictive diagnostic test for TE were calculated and receiver operating characteristic curves were obtained.

\section{RESULTS}

Clinical examination revealed that pneumothorax occurred during menses in three females with a history of pelvic endometriosis and in one with a personal history of idiopathic infertility and pleural abrasion after five recurrences. Pneumothorax occurred outside of menses in two females with a history of pelvic endometriosis.

Videothoracoscopic exploration and biopsy resulted in diagnosis of TE in nine females. Endometrial glands or chorionic cells were observed on the diaphragm in eight patients, and on both diaphragmatic and parietal pleural surfaces in one. Pathological examination of the lung resection revealed blebs in 16 patients, sarcoidosis in one and lymphangiomyomatosis in one. Surgical pleurodesis (pleural abrasion $n=29$; pleurectomy $\mathrm{n}=2$ ) was performed in all patients.

The mean \pm SD CA125 concentration of the entire population was $27.4 \pm 32.1 \mathrm{U} \cdot \mathrm{mL}^{-1}$. The level in the study group was higher than that in the control group but not significantly $\left(33.4 \pm 38.2\right.$ versus $\left.16.4 \pm 9.7 \mathrm{U} \cdot \mathrm{mL}^{-1}, \mathrm{p}=0.08\right)$. The CA125 concentration was significantly higher in females with $\mathrm{TE}$ compared to disease-free females $(76.1 \pm 49.2$ versus $16.0 \pm 8.1 \mathrm{U} \cdot \mathrm{mL}^{-1}, \mathrm{p}<0.0001$; fig. 1$)$. Figure 2 shows the receiver operating characteristic plots for CA125 measurement. The area under the curve was 0.994 . The optimum cut-off point for diagnosis of TE was $39 \mathrm{U} \cdot \mathrm{mL}^{-1}$, with no false-positive cases and two false-negative cases.

\section{DISCUSSION}

The prevalence of endometriosis can be assumed to be $\sim 10 \%$ among females of reproductive age [11]. CP is the most frequent presentation of TE, a syndrome described by JOSEPH and SAHN [12]. The other TE presentations are catamenial haemothorax, pleural effusion and catamenial haemoptysis. TE

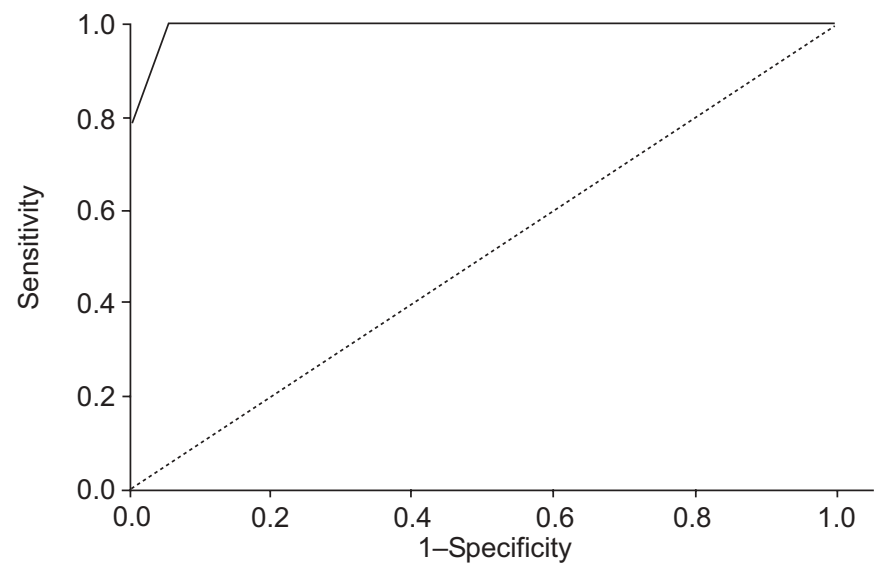

FIGURE 2. Receiver operating characteristic curve for cancer antigen 125 measurement (.......... reference line) 
is considered a rare condition. Cases of $\mathrm{CP}$ are currently published as case reports considered as very rare entities [13]. CP represents $29 \%$ (nine out of 31 ) of spontaneous pneumothorax in females. The rate observed is in agreement with rates recently published in reports prospectively studying the prevalence of TE. This rate ranges $25-33 \%[5,6]$. The mean delay between first pneumothorax and diagnosis of TE is 2.4 yrs [1].

In two-thirds (six of nine in the present population) of cases, females had a history of sterility or pelvic disease. TE frequency is perhaps underestimated because of the lack of systematic gynaecological examination. In one of the observed cases of post-operative recurrence, endometriosis was suspected by neither the gynaecologist nor the thoracic surgeon or pneumologist. The underestimated $\mathrm{CP}$ frequency may be one of the causes explaining the higher rate of recurrences in females. The most significant factor that may predispose to recurrence is sex. Females are likely to experience more recurrences than tall males [14-16].

The association between endometriosis and elevated serum CA125 levels has long been known. CA125 is expressed in amnion, derivatives of foetal coelomic epithelia and many adult tissues (endometrium, endocervix, pleura, peritoneum and pericardium) [9]. Several authors have studied CA125 concentrations during different phases of the menstrual cycle and found clearly elevated CA125 concentrations during the premenstrual late secretory phase and menstruation itself [11]. KAFALI et al. [8] recently conducted a study comparing normal females and patients with endometriosis, and observed a significantly exaggerated serum CA125 elevation in patients with endometriosis during menstruation $(\mathrm{p}<0.001)$. FEDELE et al. [17] observed that patients with advanced stages of pelvic endometriosis, according to the American Fertility Society classification [18], had serum CA125 levels significantly higher than those of disease-free females. CA125 levels in the control group of males with pneumothorax were significantly lower than observed in females with TE. The sensitivity of serum CA125 measurement in the diagnosis of endometriosis recurrence was $14.8 \%$ and the specificity was $100 \%$. Otherwise, CA125 is nonspecifically increased in any process that irritates the mesothelial cells. The most frequent pathologies that increased levels of this marker were liver cirrhosis, heart failure, serosal effusions, and pancreatic and gynaecological processes [9]. The assessment of serum CA125 level may be useful in the early diagnosis of TE in females of reproductive age presenting with primary spontaneous pneumothorax without gynaecological orientation and without clinical signs of malignant and nonmalignant conditions that injure mesothelial cells.

In summary, the present authors suggest that cancer antigen 125 measurement be included in the management of pneumothorax in females. Its elevation is a good indicator of thoracic endometriosis, as is gynaecological disease history. This biological marker may favour indication of videothoracoscopy and hormonal therapy at an early stage in the prevention of catamenial pneumothorax recurrences, and should be evaluated prospectively in a larger-scale study.

\section{REFERENCES}

1 Korom S, Canyurt H, Missbach A, et al. Catamenial pneumothorax revisited: clinical approach and systematic review of the literature. J Thorac Cardiovasc Surg 2004; 128: 502-508.

2 Maurer ER, Schaal JA, Mendez FL. Chronic recurring spontaneous pneumothorax due to endometriosis of the diaphragm. JAMA 1958; 168: 2013-2014.

3 Crutcher RR, Waltuch TL, Blue ME. Recurring spontaneous pneumothorax associated with menstruation. J Thorac Cardiovasc Surg 1967; 54: 599-602.

4 Perrotin C, Mussot S, Fadel E, Chapelier A, Dartevelle P. Pneumothorax catamenial: échec de la vidéothoracoscopie. [Catamenial pneumothorax. Failure of videothoracoscopic treatment.] Presse Med 2002; 31: 402-404.

5 Alifano M, Roth T, Camilleri Broët S, Schussler O, Magdeleinat P, Regnard JF. Catamenial pneumothorax: a prospective study. Chest 2003; 124: 1004-1008.

6 Marshall MB, Ahmed Z, Kucharczuk JC, Kaiser LR, Shrager JB. Catamenial pneumothorax: optimal hormonal and surgical management. Eur J Cardiothorac Surg 2005; 27: 662-666.

7 Tsunezuka Y, Sato H, Kodama T, Shimizu H, Kurumaya H. Expression of CA125 in thoracic endometriosis in a patient with catamenial pneumothorax. Respiration 1999; 66: 470-472.

8 Kafali H, Artuc H, Demir N. Use of CA125 fluctuation during the menstrual cycle as a tool in the clinical diagnosis of endometriosis; a preliminary report. Eur J Obstet Gynecol Reprod Biol 2004; 116: 85-88.

9 Miralles C, Orea M, Espana P, et al. Cancer antigen 125 associated with multiple benign and malignant pathologies. Ann Surg Oncol 2003; 10: 150-154.

10 Bagan P, Le Pimpec Barthes F, Assouad J, Souilamas R, Riquet M. Catamenial pneumothorax: retrospective study of surgical treatment. Ann Thorac Surg 2003; 75: 378-381.

11 Vigano P, Parazzini F, Somigliana E, Vercillini P. Endometriosis: epidemiology and aetiological factors. Best Pract Res Clin Obstet Gynaecol 2004; 18: 177-200.

12 Joseph J, Sahn SA. Thoracic endometriosis syndrome: new observations from an analysis of 110 cases. Am J Med 1996; 100: 164-170.

13 Poyraz A, Kilic D, Hatipoglu A, Demirhan B. A very rare entity: catamenial pneumothorax. Asian Cardiovasc Thorac Ann 2005; 13: 271-273.

14 Gupta D, Hansell A, Nichols T, Duong T, Ayres J, Strachan D. Epidemiology of pneumothorax in England. Thorax 2000; 55: 666-671.

15 Guo Y, Xie C, Rodriguez R, Light R. Factors related to recurrence of spontaneous pneumothorax. Respirology 2005; 10: 378-384.

16 Sadikot R, Greene T, Arnold A. Recurrence of primary spontaneous pneumothorax. Thorax 1997; 52: 805-809.

17 Fedele L, Arcaini L, Vercellini P, Bianchi S, Candiani GB. Serum CA 125 measurements in the diagnosis of endometriosis recurrence. Obstet Gynecol 1998; 72: 19-22.

18 Anon. Revised American Fertility Society classification of endometriosis: 1985. Fertil Steril 1985; 43: 351-352. 